Sackett, G. E. (1925). F. biol. Chem. 64, 203.

Salt, H. B. \& Wolff, O. H. (1957). Archs Dis. Childh. 32, 404.

Salt, H. B., Wolff, O. H., Nestadt, A. \& Lloyd, J. K. (I960). Lancet, i, 7ı.

Stone, D. B. \& Connor, N. E. (1963). Diabetes, 12, 127.

Van Eck, W. F. (1959). Am. F. Med. 27, 196.

\title{
A dietetic service for diabetics
}

\section{By Elizabeth M. Wilson, Royal Infirmary, Edinburgh, 3}

Some form of dietary regimen is required for all diabetics whatever the severity of their disorder and irrespective of whether or not insulin or an oral hypoglycaemic drug is being taken. The best results are usually obtained when the diet is simple and readily understood.

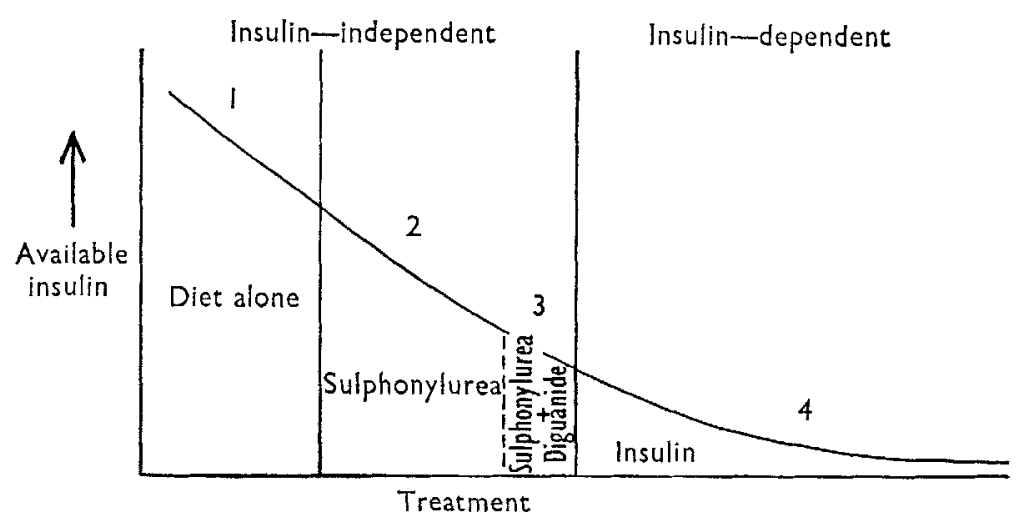

Fig. I. The treatment of diabetes in relation to the patient's ability to produce endogenous insulin.

\section{Classification of diabetes}

Fig. I shows a scheme for the treatment of diabetes in relation to the patient's ability to produce endogenous insulin. If the amount of available insulin is large, treatment is by diet alone; if moderate, it is by diet plus an oral hypoglycaemic agent, and if little or no insulin is being secreted, by diet plus insulin. Diabetics in groups I, 2 and 3 are therefore insulin-independent and those in group 4 insulin-dependent. The diagram can also be interpreted by taking the line of demarcation between 3 and 4 as the age 40 . As a general rule, patients diagnosed to have diabetes before the age of 40 are insulin-dependent and those older than this at diagnosis are insulinindependent.

\section{Principles of diets for diabetics}

Diets for diabetics should conform to the following principles: ( $\mathrm{I}$ ) The avoidance of all forms of concentrated carbohydrate. Simple sugars are quickly absorbed into the bloodstream and cause a rapid postprandial increase in glycaemia, which is difficult to control and may lead to overdosing with insulin. (2) The restriction of, or the prescription in definite quantities of, other forms of carbohydrate. Polysaccharides are more slowly absorbed than sugars, giving a gradual increase 
in the blood sugar level, which can be more readily controlled. (3) No limitation of protein. (4) No restriction of fat, except in the case of obese diabetics.

Calories. Unless obese, a diabetic needs the same number of calories as a nondiabetic of the same age, sex and occupation. In fact, underweight patients will often require a greater calorie intake.

Carbohydrate. In the days before insulin discovery, diabetics were fed on extremely low carbohydrate diets, which were very unpalatable owing to the high amount of fat necessary to make up the calories. The modern trend is to give a larger and more adequate carbohydrate intake, usually between $200-240 \mathrm{~g}$ daily; but obese patients may be given $\mathrm{I} 20 \mathrm{~g}$ whereas a hard-working labourer may require $280-320 \mathrm{~g}$ of carbohydrate daily.

Protein. Protein should be adequate to meet requirements of growth in children and young adults. In the elderly, care should be taken to ensure that sufficient protein is taken.

Fat. Fat requires to be restricted only in the obese diabetic. It is debatable whether animal fat should be limited, if, as is thought, diabetics are more prone to develop atherosclerotic vascular disease at an earlier age than the general population. The serum cholesterol level is often raised in diabetes, but as endogenous metabolism of cholesterol is independent of dietary intake, there is no purpose in deliberately restricting cholesterol-containing foods.

Other factors. In addition to the above principles the diet should: (I) Be simple to follow otherwise it will be useless. All too often, patients are furnished with several sheets of instructions which are so complex that they cannot be understood and are in consequence quickly abandoned. (2) Be suited to the patient's age, sex, occupation and social conditions. The young need more calories than the old; men allegedly need more calories than women; a miner working underground needs more calories than a sedentary worker; and a man living in a model lodging house, where communal feeding is the order of the day, needs a type of diet quite different to that of the society lady. (3) Not involve special cooking or unnecessary expense. A brief diet history of the patient's previous meal pattern should be taken and, where possible, the diet made to fit in with the family meal pattern. If a packed or canteen lunch is taken, this should provide no difficulty and as no special foods are required by diabetics the family menus can be easily adapted. (4) Not precipitate any nutritional deficiency.

\section{Types of diets for diabetics}

Simple carbohydrate restriction. This type of diet is suitable for elderly or middleaged mild diabetics, treated by diet alone or with an oral hypoglycaemic drug, where rigid control is unnecessary and often undesirable. The aim is to abolish symptoms and keep the overnight urine generally free of sugar. The necessary restriction is encouraged by three lists of foods: (a) all concentrated carbohydrates which are to be avoided; (b) protein-, fat- and low-calorie-containing foods which can be taken without restriction; (c) other forms of carbohydrate which can be taken in moderate quantities only. 
Carbohydrate exchange system in which the exchanges are given in terms of domestic measures. This diet is suitable for (a) moderately severe diabetics, who are controlled by oral hypoglycaemic agents and who fail to respond to simple carbohydrate restriction, (b) obese diabetics, (c) insulin-dependent diabetics, who are unable to follow a weighed diet owing to age, failing sight, low intellect or social circumstances. It also consists of three lists: (a) concentrated carbohydrate to be avoided completely; (b) protein and low-calorie foods to be consumed without limit; (c) other carbohydrate foods to be consumed in limited amounts each equal to approximately I $5 \mathrm{~g}$ carbohydrate. In the list suggested amounts are given in 'helpings' and 'slices'. An allowance of milk and fat can be given if the calorie intake is to be controlled as, for example, in the obese.

Weighed carbohydrate exchange system. This diet is suitable for young and middleaged insulin-dependent diabetics. It consists of unlimited protein, fat and lowcalorie foods and a list is presented of starchy foods each equivalent to $15 \mathrm{~g}$ carbohydrate.

Table I shows that a diet of $240 \mathrm{~g}$ carbohydrate would consist of I pint of milk and fourteen portions of choice or 'exchanges', the distribution of the exchanges throughout the day being dependent on the type or types of insulin being taken. It is essential that the same number of exchanges be taken at the corresponding meal of each day. By using this weighed exchange diet, patients can get variety in their diets and can take in their stride canteen meals, school lunches, business lunches, picnics, dinner dances and even banquets.

Table I. Calculation of diabetic diet

\begin{tabular}{lc}
\multicolumn{2}{c}{ (e.g. $240 \mathrm{~g} \leftarrow$ carbohydrate) } \\
& Carbohydrate provided \\
& $30 \mathrm{~g}$ \\
I pint milk & $210 \mathrm{~g}$ \\
Fourteen 'exchanges'* & $240 \mathrm{~g}$ \\
$14 \times 15$ g portions & Total \\
*One 'exchange' portion provides $15 \mathrm{~g}$ carbohydrate.
\end{tabular}

During illness, it is important that as nearly as possible the full number of carbohydrate exchanges is taken and extra carbohydrate may have to be taken to compensate for the increased glycosuria. A list of easily digested forms of carbohydrate should be given during illness; too sweet foods should be avoided as these often lead to vomiting and an increase in dehydration and ketosis.

\section{Alcohol}

Alcohol raises a problem which worries many diabetics. Sweet wines, sweet sherries, liqueurs, beers and stouts should be avoided by all diabetics. Spirits may be taken, except by the obese, but care should be taken by patients receiving oral therapy, as this sometimes causes an increased sensitivity to alcohol.

\section{Summary}

Success in the treatment of diabetes can be achieved providing time is initially spent in instructing the patient in the type of diet most suited to his intelligence, 
social conditions, previous dietary habits and the type of diabetes and its therapy. Patients furnished with a highly and unnecessarily complex dietary system usually abandon any attempt to adhere to it, with resultant loss in diabetic control.

\section{Diet and the development of clinical diabetes}

By Joyce D. Baird and M. Kellock, Western General Hospital, Edinburgh Text for publication not received.

\section{Proprietary foods for diabetics}

By T. D. Kellock, 4r Harley Street, London

Text for publication not received. 